An Iterative Method for Electrostatic Object Reconstruction in a Half Space

van Berkel, Cees and Lionheart, William R. B.

2005

MIMS EPrint: 2005.51

Manchester Institute for Mathematical Sciences

School of Mathematics

The University of Manchester

\footnotetext{
Reports available from: http://eprints.maths.manchester.ac.uk/

And by contacting: The MIMS Secretary

School of Mathematics

The University of Manchester

Manchester, M13 9PL, UK
} 


\title{
An Iterative Method for Electrostatic Object Reconstruction in a Half Space
}

\author{
Cees van Berkel $\dagger$ and William R B Lionheart $\ddagger$ \\ $\dagger$ Philips Research Laboratories, Redhill RH1 5HA, UK \\ †School of Mathematics, The University of Manchester, PO Box 88, Sackville Street Manchester M60 \\ $1 \mathrm{QD}, \mathrm{UK}$ \\ E-mail: cees.van.berkel@philips.com
}

\begin{abstract}
Sensing electrodes arranged in or around a display can provide input function for interactive displays. Commercially this is interesting because the sensing electrodes and electronics can be made in the same manufacturing process as that of the display itself thus reducing cost. In engineering terms the electrodes measure capacitance changes resulting from the presence and movement of objects such as hands and fingers in front of the display. At the quasi static frequencies used $(100 \mathrm{kHz})$ the human body is conductive and the hands or fingers provide a screen between the capacitive electrodes. There is no need to touch the actual display and the overall system constitutes a touchless gesture input system.

Determining the shape of the hand or fingers is a boundary condition reconstruction problem of finding the boundary of an earthed conductive object $D$ from electrostatic measurements. This is the ill-posed problem of recovering the zero-surface of a solution to Laplace's equation from Cauchy data on part of the boundary of a domain. The problem has similarities with object reconstruction in EIT or inverse scattering but is complicated because only a partial Dirichelet-Neumannn map is available as experimental data.

We suggest an algorithm where at each iteration we have an approximation $\partial D_{k}$ to $\partial D$ on which we calculate approximate Cauchy data by solving a Tikhonov regularized linear system. This data is used to modify $\partial D_{k}$ by extrapolation towards the zero-surface giving the next approximation $\partial D_{k+1}$.

We implemented the algorithm in two and three space dimensions using the Boundary Element Method for discretization. Numerical results using simulated data with added noise show that simply connected but not necessarily convex objects can be reconstructed with reasonable positional accuracy and approximate shape, but as might be expected the shape is more accurately determined near the plane of measurements.
\end{abstract}

Submitted to: Meas. Sci. Technol.

\section{Introduction}

Consider a grounded and conductive object in the half space $H:=\left\{x \in \mathbb{R}^{3}: x_{3}>0\right\}$ above an infinite plane $\partial H$. Cauchy data are obtained from capacitance measurements made between electrodes in the plane with the aim to establish the location, size and shape of the object. We thus have the problem of finding a domain $D \subset H$ with smooth boundary $\partial D$, such that

$$
\nabla^{2} u=0 \quad \text { in } H \backslash \bar{D}
$$

where

$$
\begin{array}{ll}
u=f_{H} & \text { on } \partial H \\
\frac{\partial u}{\partial n}=g_{H} & \text { on } \partial H \\
u=0 & \text { on } \partial D
\end{array}
$$

In this $f_{H}$ is the known potential distribution on the plane and $g_{H}$ the measured charge distribution on the plane. $n$ is the unit normal pointing out of the domain $H \backslash \bar{D}$.

This problem is of interest in providing pointing and gesture input to display screens [1]. The display is regarded as a ground plane and quasi-static measurements are made between electrodes arranged in or around the display. The measurements are performed at a frequency high enough to regard the human body as conductive and connected to ground, but low enough to justify an electrostatic formulation [2]. 

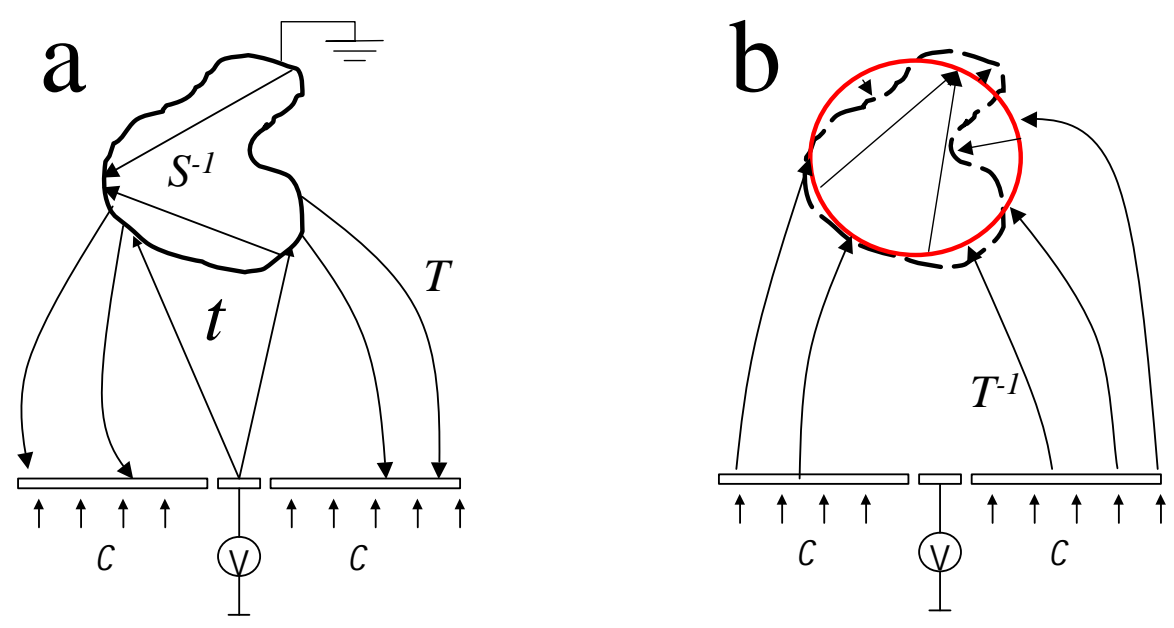

Figure 1. A) Schematic illustration of cross capacitance charge imaging. B) Schematic illustration of the cross capacitance object reconstruction.

Here we study a simplified and idealized version of the problem. Our aim is to show that it is numerically feasible to extract shape information from limited and noisy data. We require to do this specifically in the $3 \mathrm{D}$ setting. Note that the $1 \mathrm{D}$ version of the problem is trivial; it corresponds to finding the separation $d$ of the parallel plate capacitance. For the 2D case, the logarithmic fundamental solution limits the relevance to the practical situation of finite $3 \mathrm{D}$ objects and sensors arrangements. The next section describes the forward problem and introduces the concept of a charge image: The change in charge distribution on the plane $\partial H$ resulting from the presence of the object. It is an image in sense that it contains enough information to recover ('see') the shape of the object. Section 3 formulates the inverse problem and explores the relation with other, better known inverse problems. Sections 4 to 6 set out our solution to the inverse problem. Numerical examples are then provided in the following sections.

\section{FORWARD PROBLEM}

This section gives an integral formulation of the forward charge imaging problem. We consider the case of a single voltage electrode, referred to as the transmitter, at the origin. As illustrated in Figure 1A, a grounded object is located above the ground plane. Using Green's theorem, the potential in the space above the sensor plane is given by

$$
u(x)=\int_{\partial H} f_{H}(y) \frac{\partial G(x, y)}{\partial y_{3}} \mathrm{~d} s(y)+\int_{\partial D} G(x, y) g_{D}(y) \mathrm{d} s(y), \quad x \in H \backslash \bar{D}
$$

$g_{D}=\partial u / \partial n$ is the charge distribution on the object. $G(x, y)$ is the free space Green's function including the image charge contribution to account for the ground plane $[3,4]$. This means that there is no single layer contribution on $\partial H$. Also, the fact that $u(x)=0$ on $\partial D$ was used to ignore the double layer potential contribution on $\partial D$. The potential distribution $f_{H}(y)$ on the plane will typically be nonzero only for the small, central transmitter electrode. The first term in (1) represents the contribution to $u(x)$ from the transmitter, $y_{3}$ represents the coordinate along the positive $x_{3}$-axis, that is, opposite to the outward pointing normal. The second term in (1) is the contribution to the potential from the charge distribution $g_{D}$ on the object. The charge distribution $g_{D}$ is found by solving (1) with $u=0$ on $\partial D$.

$$
-\int_{\partial D} G(x, y) g_{D}(y) \mathrm{d} s(y)=\int_{\partial H} f_{H}(y) \frac{\partial G(x, y)}{\partial y_{3}} \mathrm{~d} s(y), x \in \partial D
$$

Although the integral operator on the LHS is compact, the equation is solved numerically with relative ease. This is due to the smoothness of the RHS and the fact that as an operator from $H^{-1 / 2}(\partial D) \rightarrow$ $H^{1 / 2}(\partial D)$, the operator has a bounded inverse [5].

The presence of the object decreases the charge density on the ground plane. We refer to this decrease as a charge image $c(x)$. It is given by the normal derivative of the second term in (1).

$$
c(x)=-\int_{\partial D} \frac{\partial G(x, y)}{\partial x_{3}} g_{D}(y) \mathrm{d} s(y), x \in \partial H
$$




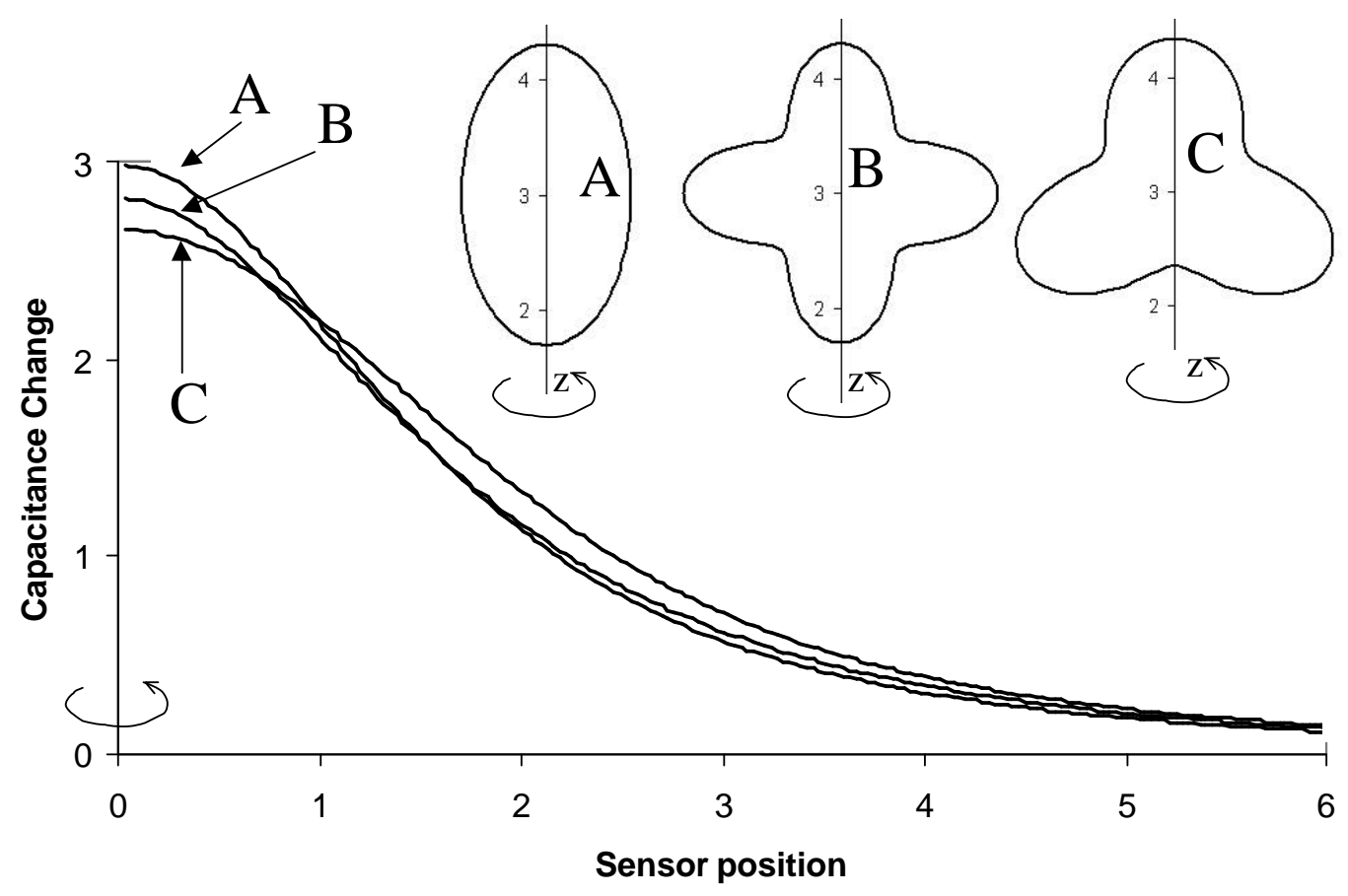

Figure 2. Simulated charge image $c(x)$ for three axi-symmetric objects. Indicated is the axis of rotation and the shape of the three objects used.

The minus sign in front of the integral is due to the fact that $x_{3}$ is oriented opposite to the outward pointing normal. $c(x)$ is measured as a decrease in capacitance between the transmitter and electrodes in the ground plane.

Figure 2 illustrates the simulated charge image $c(x)$ for a number of on axi-symmetric 3D objects. Using rotational symmetry has the advantage of computational efficiency as well as representational expediency while at the same time remaining close, in terms of the Green's function for example, to the full 3D situation that we are ultimately interested in. For clarity, images are plotted noise free, although in the reconstructions below, $1 \%$ or $10 \%$ white noise is added. The shape of the three objects is illustrated in the figure.

\section{THE INVERSE PROBLEM}

As can be seen the charge images for the three different shapes are different, but it is not immediately clear that these differences are enough to distinguish between them or even reconstruct the different shapes from the charge images alone. It is to this inverse problem that we now turn. The challenge is to find the domain $D$ from limited and noisy measurements of $c(x)$. That is from Cauchy data on the plane $\partial H$. These measurements are referred to as cross capacitance sensing.

The problem is solved by identifying $\partial D$ as the zero potential contour of the unique solution of the Cauchy problem for the Laplace equation. A substantial literature exists on inverse boundary problems of this type, mainly on bounded domains in 2D. For instance, Akduman and Kress [6] studied 2D shape reconstruction from Cauchy data on an enclosing annulus. Hähner [5] used Dirichlet data on an enclosing open ball for a multitude of sources for reconstruction of 3D shapes. The operator properties considered there are relevant to the analysis of eqns (1) and (2) here. Some approaches to Electrical Impedance Tomography (EIT) [7] and crack detection $[8,9]$ also tackle inverse boundary problems for the Laplace equation. In 2D, conditional logarithmic stability estimates for the inverse boundary problem under a regularity assumption on the unknown boundary have been given $[10,11]$, and this theoretical result has been extended to 3D by Cheng et al. [12].

The problem here must be distinguished from that in Electrical Capacitance Tomography (ECT) [13], which also measures the capacitance between points, but seeks to find domains of differing dielectric permittivity and is an inverse coefficient problem. It is also important to point out that there are capacitance measurements in which the load capacitance to single electrodes is measured $[14,15,16]$. These techniques also aim to create an 'image' of a remote object but in the vocabulary of this paper, 
this image is the sum of multiple $c(x)$, each one sampled at the just point, the position of the corresponding transmitter. The solution in this paper does not directly provide a reconstruction for this case, but we look forward to extending the work in the future. As an applied inverse problem, finding $D$ from $c(x)$ has much in common with inverse acoustic scattering where single layer potential expressions similar to eqn. (3) are found.

\section{INVERSE CHARGE IMAGING}

In this paper we use an iterative approach to the inverse problem. A reconstruction algorithm using an indicator function is described elsewhere[17]. The iteration is illustrated in Figure 1B. At each step $k$, we first use the data $c(x)$ to find a potential and charge distribution on an object $\partial D_{k}$, the current guess. This is used to deform $\partial D_{k}$ towards the real object, yielding $\partial D_{k+1}$. The process is then repeated. This section deals with problem of finding a potential and charge distribution on the object $\partial D_{k}$.

We make the assumption that we have a current guess $\partial D_{k}$ which encloses all singularities of the analytic continuation of $u$. An initial guess $\partial D_{0}$ can be obtained using closed form expressions for the charge image of a sphere with uniform charge distribution in a Gauss-Newton optimization for position and size. We also refer to Kim et al. [18] for an interesting example of object location on a bounded domain. Writing $f_{D_{k}}$ for the potential on the guess and $g_{D_{k}}$ for the charge distribution, we obtain from Green's theorem

$$
\begin{aligned}
-u(x) & =-\int_{\partial H} f_{H}(y) \frac{\partial G(x, y)}{\partial y_{3}} \mathrm{~d} s(y)+\int_{\partial D_{k}} f_{D_{k}}(y) \frac{\partial G(x, y)}{\partial n(y)} \mathrm{d} s(y) \\
& -\int_{\partial D_{k}} G(x, y) g_{D_{k}}(y) \mathrm{d} s(y), \quad x \in H \backslash \bar{D}_{k}
\end{aligned}
$$

Because the assumption that $u(x)=0$ does not hold on $\partial D_{k}$, the double layer potential contribution on $\partial D_{k}$ has now been included.

We can evaluate eqn. (4) at the boundaries to obtain a system of equations for the boundary conditions. Specifically we evaluate (4) on the boundary of the object to find an equation for $f_{D_{k}}$. Here we need to introduce the factor $1 / 2$ to account for the evaluation on the boundary. The normal derivative of (4) on the ground plane is used to obtain an equation for $g_{H}$. Hence

$$
\begin{aligned}
-\frac{1}{2} f_{D_{k}}(x) & =-\int_{\partial H} f_{H}(y) \frac{\partial G(x, y)}{\partial y_{3}} \mathrm{~d} s(y)+\int_{\partial D_{k}} f_{D_{k}}(y) \frac{\partial G(x, y)}{\partial n(y)} \mathrm{d} s(y) \\
& -\int_{\partial D_{k}} G(x, y) g_{D_{k}}(y) \mathrm{d} s(y), \quad x \in \partial D_{k} \\
g_{H}(x) \quad & -\int_{\partial H} f_{H}(y) \frac{\partial^{2} G(x, y)}{\partial y_{3} \partial x_{3}} \mathrm{~d} s(y)+\int_{\partial D_{k}} f_{D_{k}}(y) \frac{\partial^{2} G(x, y)}{\partial x_{3} \partial n(y)} \mathrm{d} s(y) \\
& -\int_{\partial D_{k}} \frac{\partial G(x, y)}{\partial x_{3}} g_{D_{k}}(y) \mathrm{d} s(y), \quad x \in \partial D_{k}
\end{aligned}
$$

Introducing the following operator short hand

$$
\begin{aligned}
v(x) & =f_{D_{k}}(x) \\
q(x) & =-g_{D_{k}}(x) \\
t(x) & =\int_{\partial H} f_{H}(y) \frac{\partial G(x, y)}{\partial y_{3}} \mathrm{~d} s(y) \\
(K v)(x) & =\int_{\partial D_{k}} f_{D_{k}}(y) \frac{\partial G(x, y)}{\partial n(y)} \mathrm{d} s(y) \\
(S q)(x) & =-\int_{\partial D_{k}} G(x, y) g_{D_{k}}(y) \mathrm{d} s(y) \\
c(x) & =\int_{\partial H} f_{H}(y) \frac{\partial^{2} G(x, y)}{\partial y_{3} \partial x_{3}} \mathrm{~d} s(y)-g_{H}(x) \\
(M v)(x) & =\int_{\partial D_{k}} f_{D_{k}}(y) \frac{\partial^{2} G(x, y)}{\partial x_{3} \partial n(y)} \mathrm{d} s(y) \\
(T q)(x) & =-\int_{\partial D_{k}} \frac{\partial G(x, y)}{\partial x_{3}} g_{D_{k}}(y) \mathrm{d} s(y)
\end{aligned}
$$


The equations become

$$
\left(\begin{array}{cc}
\frac{1}{2} I+K & S \\
M & T
\end{array}\right)\left(\begin{array}{l}
v \\
q
\end{array}\right)=\left(\begin{array}{c}
t \\
c
\end{array}\right)
$$

Here the Cauchy data on the object $(v, q)$ is mapped to the functions $(t, c)$ which are derived from the Cauchy data on the sensor plate. The inverse problem of finding $(v, q)$ from $(t, c)$ is the ill-posed problem of finding Cauchy data on a part of the boundary.

It is therefore necessary to use regularisation to solve $(5)$ to find $(v, q)$ from $(t, c)$. The regularisation is provided by the knowledge that $\partial D_{k}$ is close to the real object and the potential on $\partial D_{k}$ is small. The norm $\|v\|$ must therefore be small and this requirement is added to the solution to (5). Hence $v, q$ are found through minimization of the Tikhonov functional:

$$
\left\|\frac{1}{2} v+K v+S q-t\right\|^{2}+\|M v+T q-c\|^{2}+\alpha_{v}^{2}\|v\|_{P}^{2}
$$

In which $\|\cdot\|_{P}$ is a first order Sobolev norm and $\alpha_{v}$ is the Tikhonov regularisation parameter. In numerical experiments we have found that minimizing this functional works well to recover approximate Dirichlet and Neumann data on a guess $\partial D_{k}$. In the next section we will discuss how this data can be used to deform the object $\partial D_{k}$ towards an improved approximation $\partial D_{k+1}$.

We also consider a simplified functional

$$
\|v+S q-t\|^{2}+4\|T q-c\|^{2}+\alpha_{q}^{2}\left\|q-q_{p}\right\|_{P_{Q}}^{2}
$$

In this $K v$ and $M v$ terms are approximated with $\frac{1}{2} v$ and $q T-c$ respectively. This substitution is motivated by numerical efficiency. No $K$ and $M$ matrices have to be calculated and the first term in the functional can be minimized independently of the last two. It is validated by the results shown in the next section. The simplification can be justified by the fact that as $\partial D_{k}$ approaches the correct $\partial D$, the variation in $f_{D_{k}}$ on $\partial D_{k}$ becomes small. Using proposition 3.19 of [19] we can approximate

$$
(K v)(x) \approx f_{D_{k}}(x) \int_{\partial D_{k}} \frac{\partial G(x, y)}{\partial n(y)} \mathrm{d} s(y)=\frac{1}{2} v(x)
$$

At the same time, it can be shown that in the second term $q T-c \approx M v$ as $\partial D_{k} \rightarrow \partial D$. The second term in (7) is independent of $v(x)$ and alternative regularisation is required. We now require that the charge distribution $q$ on $\partial D_{k}$ is close to a charge distribution corresponding to $v(x)=0$ on $\partial D_{k}$. This implies a prior for the charge distribution that satisfies eqn.(2) on $\partial D_{k}$, that is $q_{p}(x)=\left(S^{-1} t\right)(x)$.

Moreover, the prior needs to measured by a normalized first order Sobolev norm $\|\cdot\|_{P_{Q}}$ in which $P_{Q}=Q_{p}^{-1} P Q_{p}^{-1}$ and

$$
\left(Q_{p}^{-1} f\right)(x)=\frac{f(x)}{q_{p}(x)} \quad x \in \partial D_{k}
$$

The reason for the use of the normalisation operator $Q_{p}^{-1}$ is that $q_{p}(x)$ will vary by several orders of magnitude over an object and will be smallest on the parts of $\partial D_{k}$ furthest away from the transmitter and sensor plate. It there where the instability in $q(x)$ is greatest and a pre-conditioned penalty term is required to constrain solution there. Another way of interpreting the use of the $Q_{p}^{-1} P Q_{p}^{-1}$ norm is that in a statistical sense the prior has a covariance $Q_{p}^{2}$ and an expected value of $q_{p}(x)[20]$.

\section{OBJECT RECONSTRUCTION}

Having obtained the potential and charge distribution we now seek to deform the current guess towards the real object guided by the knowledge that this real object is at ground potential. One method that readily suggests itself is to use eqn.(4) to find a zero contour, or at least a minimum contour, near the object and identify that as our next best guess. This approach has been reported for acoustic scattering [21, 22]. A variation on this would be to refrain from calculating the actual zero contour but use the potential $v$ and the gradient $q$ to make a extrapolation from $\partial D_{k}$ towards the zero potential contour.

In numerical experiments we have found that neither method works well. The reason is that finding or extrapolating towards the zero potential contour will induce the strongest changes in $\partial D_{k}$ where $q(x)$ is smallest, i.e. those parts for which the conditioning of eqn.(5) is worst. We therefore opt for an extrapolation method which is pre-conditioned with the operator $Q_{p}$. For linear extrapolation this leads to a dilation function $h(x)$ which gives the deformation at $x$ on the object along the outward pointing normal.

$$
h(x)=-\lambda v(x) \quad x \in D_{k}
$$



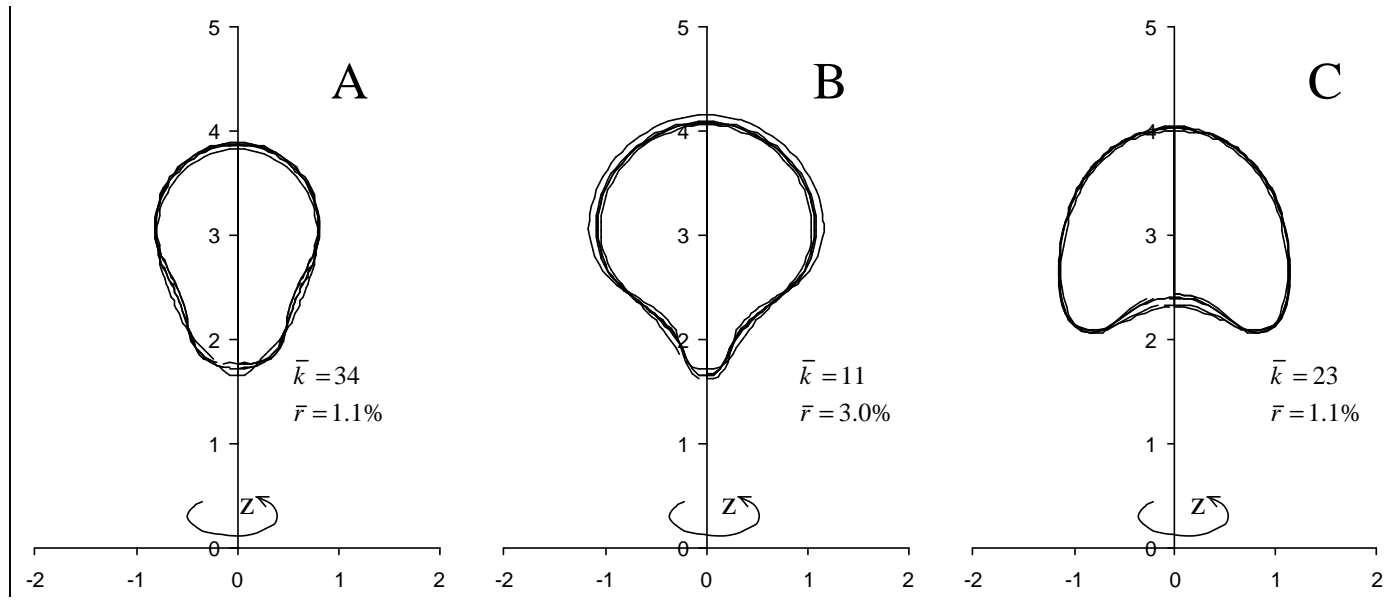

Figure 3. Reconstructions from the charge images in Figure 2 using functional (6). The average iteration number $(\bar{k})$ and residue at termination $(\bar{r})$ are shown in insets.

The attenuation, or relaxation, factor $\lambda$ avoids overshoot and it is a second, independent regularisation parameter.

\section{STOPPING CRITERION}

We thus have a cross capacitance reconstruction algorithm in which the shape is found from a level set evolution driven by the potential $u$ itself. Level sets have been used in a variety of shape reconstruction problems $[23,24,25]$. Briefly stated the algorithm here starts with an initial guess and then, through repeated application of either eqns (6) and (8), or eqns (7) and (8), evolves the object until a stopping criterion is reached.

A common method for deciding when a best fit has been obtained is the so called 'Morozov Discrepancy Principle', which states that a good fit is obtained when the difference between measured and fitted image, the so called cost function, is similar to the noise in the measured image. A drawback of this criterion is however that it requires prior knowledge of the noise in the measurements and is in any case only valid for true white noise. Instead we use the change in cost function for our stopping criterion. Thus the iteration is stopped when the iteration to iteration change in the cost function falls below a certain threshold.

$$
\left|\frac{r_{k}-r_{k-1}}{r_{k}}\right|<E
$$

In which $E$ is the threshold. The cost function, or residue, $r_{k}$ at iteration $k$ is defined in terms of the piecewise constant elements of the charge image used in the numerical experiments.

$$
r_{k}=\sqrt{\frac{1}{n} \sum_{i=1}^{n}\left(\frac{c_{i}^{k}-c_{i}}{c_{i}^{k}}\right)^{2}}
$$

In which $c_{i}$ represents the $i$-th piecewise constant element in the noisy input image and $c_{i}^{k}$ the $i$-th element of the charge image of the current reconstruction. Strictly speaking the stopping criterion is only sensible and can only be guaranteed to stop the iteration if $r_{k}$ is strictly decreasing with iteration number. Currently we lack a formal proof for this, but a strictly decreasing behaviour in the numerical experiments is always observed. The threshold $E$ is an additional regularisation parameter. Choosing $E$ too large has obvious drawbacks, but also at excessively small values poor reconstruction is obtained. We have found that a value of $E=0.01$ worked well in all numerical experiments. We stress that with this construction, the actual noise level is not an input parameter to the stopping criterion.

\section{PARAMETER SCALING}

Before we turn to the numerical results in the next section a refinement to eqns (6)-(8) is made by introducing scaled regularisation parameters $\hat{\alpha}$ and $\hat{\lambda}$ that are insensitive to the scale and discretization 

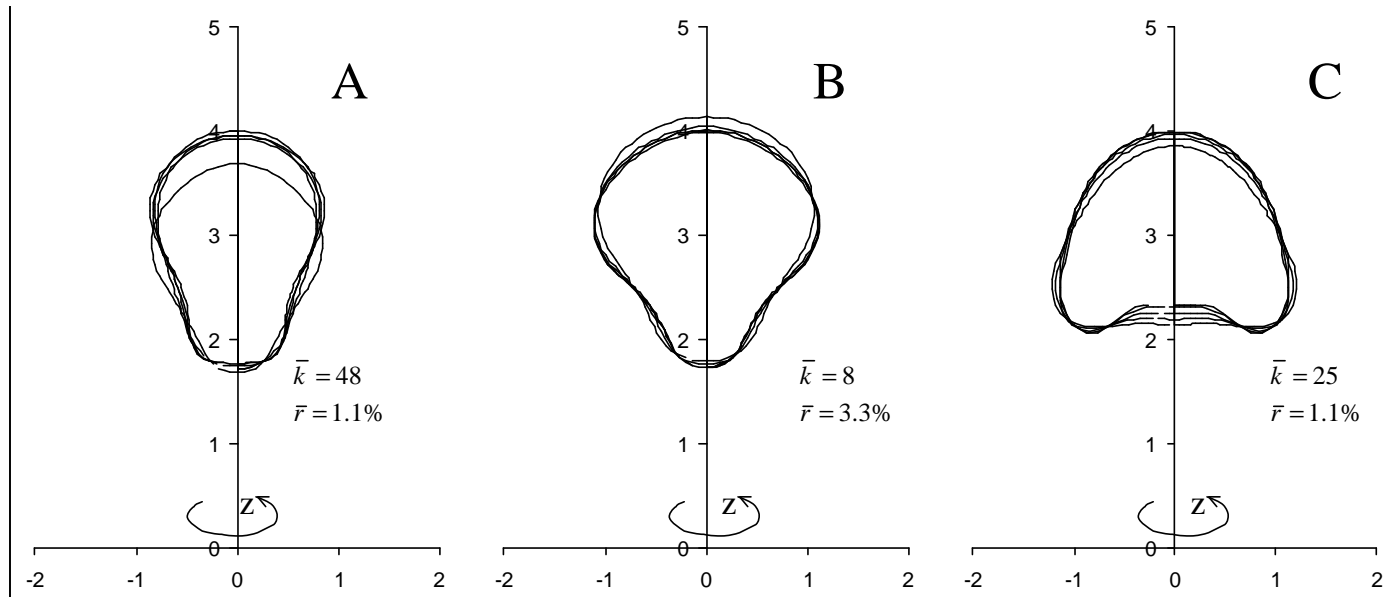

Figure 4. Reconstructions from the charge images in Figure 2 using functional (7). The average iteration number $(\bar{k})$ and residue at termination $(\bar{r})$ are shown in insets.

level and can be used in either functional (6) or (7)

$$
\begin{aligned}
& \alpha_{v}=\hat{\alpha} \frac{\|M\|}{\sqrt{\|P\|}} \\
& \alpha_{q}=\hat{\alpha} \frac{\|T\|}{\left\|Q_{p}^{-1}\right\| \sqrt{\|P\|}} \\
& \lambda=\frac{\hat{\lambda}}{\left\|Q_{p}\right\|}
\end{aligned}
$$

These factors allow comparison of the effectiveness of the penalty terms in functionals (6) and (7). The factors $\hat{\alpha}$ and $\hat{\lambda}$ are used as input to the cross capacitance object reconstruction and the values of $\lambda, \alpha_{v}$ or $\alpha_{q}$ and are then calculated once only, using the initial guess to calculate the operator norms.

\section{NUMERICAL RESULTS}

Numerical simulations were performed on a personal computer using $\mathrm{C}++$ code with NAG library support. The simulations are calculated using a Boundary Element Method (BEM) formulation [26, 27] with piecewise constant $v, q, t$ and $c$. The Cauchy principal value integral is used to remove the singularity in the $S$ and $K$ integral operators. One variant of the code was implemented for an axi-symmetric problem in which the object and the sample points of $c(x)$ have rotational symmetry around the $x_{3}$-axis. This reduces the problem to a two dimensional one in which the Greens function $G(x, y)$ and its derivative on the ground plane are expressed in complete elliptic integrals of the first and second kind [28]. The second variant of the code implemented the full 3D problem for non-symmetric triangulated wire frame models of the objects.

Figure 3 illustrates reconstructions using functional (6) for the axi-symmetric objects of the (axisymmetric) charge images illustrated in Figure 2. Each object was reconstructed five times for different draws of $1 \%$ normally distributed noise added to the input image. The objects were defined in 51 linear line segments and the charge image $c(x)$ was sampled at 200 equidistant radial points from $\rho=0$ to $\rho=6$. In each case a unit sphere centred at $x_{3}=3$ was used as initial guess. Heuristic regularisation parameters $\hat{\alpha}=1$ and $\hat{\lambda}=1$ were used.

The objects and reconstructions show the variation in reconstruction attributable to the noise in the input images and the reconstructions here have been chosen to illustrate both what can be and what cannot be reconstructed. As is perhaps obvious, no significant reconstruction is achieved on the side of the object facing away from the sensor plane. However, fair reconstruction is obtained at the facing side.

Figure 4 illustrates reconstructions using simplified functional (7) for the same axi-symmetric objects. Again each object was reconstructed five times for different draws of $1 \%$ Gaussian noise added to the input image and regularisation parameters $\hat{\alpha}=1$ and $\hat{\lambda}=1$ were again used. As can be seen, the reconstructions are very similar to those shown in Figure 3. These reconstructions typically took a third of the CPU time of the reconstructions shown in Figure 3. We stress that the same $\hat{\alpha}$ and $\hat{\lambda}$ were used 

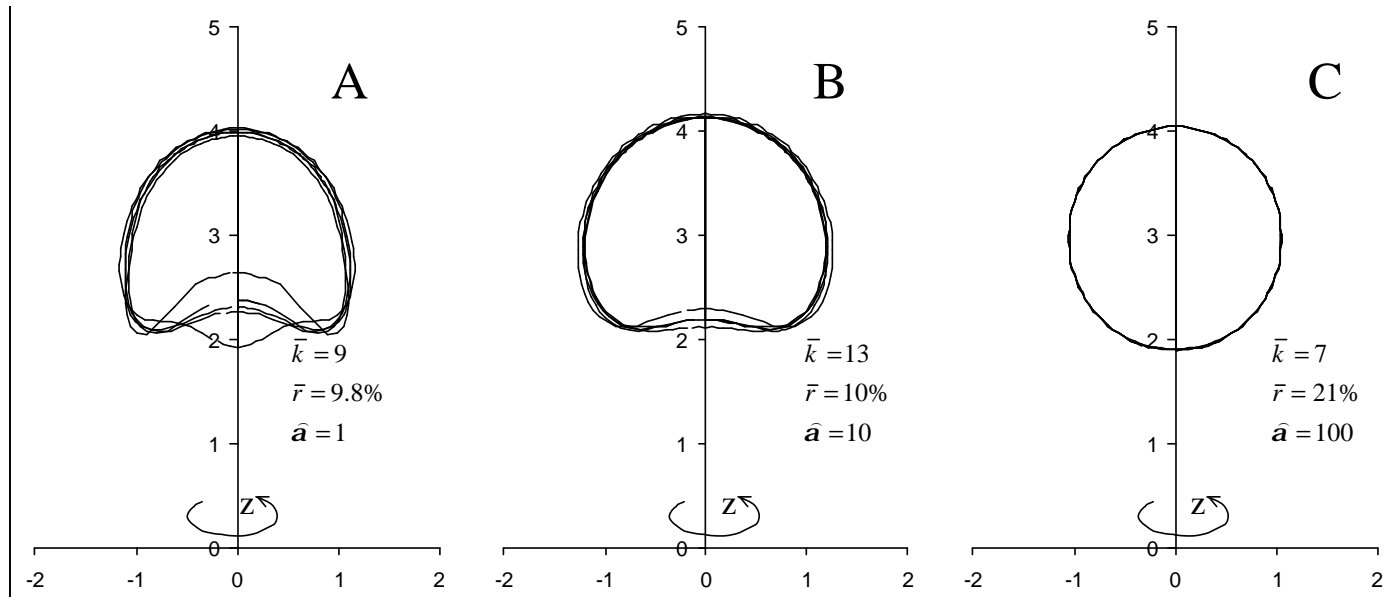

Figure 5. Reconstructions at $10 \%$ input noise level for different regularisation parameters using functional (6). The average iteration number $(\bar{k})$ and residue at termination $(\bar{r})$ and the regularisation parameter $(\hat{\alpha})$ are shown in insets.

for the results in Figures 3 and 4. The actual regularisation parameters $\alpha_{v}$ and $\alpha_{q}$ used in functionals (6) and (7) differed by two orders of magnitude. Results at $3 \times$ discretization or $100 \times$ scale, again with the same $\hat{\alpha}$ and $\hat{\lambda}$, but with very different values of $\alpha_{v}, \alpha_{q}$ and $\lambda$, yield virtually identical results. These observations support the approximate equivalence of the functionals and the scaling of the regularisation parameters.

The results in Figure 5 explore the effect of the input noise and the Tikhonov regularisation parameter $\hat{\alpha}$ on the reconstruction. Shown are three reconstructions of object $\mathrm{C}$ in Figure 2, each again for 5 draws of the input noise, which is this time set at $10 \%$ rather that $1 \%$. The reconstruction in Figure $5 \mathrm{~A}$ was done with the same regularisation parameters as before $(\hat{\alpha}=1, \hat{\lambda}=1)$ and shows that increased noise on the input data deteriorates the reconstruction. This deterioration is particularly noticeable as an increased variation in the reconstructed object between noise draws. In Figure $5 \mathrm{~B}$ this variation is reduced by increasing the Tikhonov regularisation parameter $(\hat{\alpha}=10, \hat{\lambda}=1)$. Figure $5 \mathrm{C}$ shows that increasing the regularisation parameter further $(\hat{\alpha}=100, \hat{\lambda}=1)$ reduces the variation to zero but also obliterates meaningful reconstruction. Note that the average error at termination is close to the $10 \%$ input noise level for all results in Figure 5.

\section{MULTIPLE TRANSMITTERS}

In the situation of practical interest the experimental data are obtained from a linear array of electrodes arranged around the edge of a display surface, instead of being distributed over the whole surface of the display itself. Also, each electrode in turn functions as a transmitter electrode, collecting $n$ seperate charge images $c_{i}$. This influences the object reconstruction in a number of ways. On the one hand the reduced aperture of the measurements will make the problem harder. On the other hand, the multiple charge images $c_{i}$ provide a richer data set in analogy with multiple incident wave directions in acoustic scattering[21] or multiple illumination sources in machine vision.

Now (5) must be solved for each transmitter $i$, using simplified functional (7), we obtain the sum

$$
\sum_{i}\left\|v_{i}+S q_{i}-t_{i}\right\|^{2}+4\left\|T q_{i}-c_{i}\right\|^{2}+\alpha_{q}^{2}\left\|q_{i}-q_{p, i}\right\|_{P_{Q, i}}^{2}
$$

In which the sum is over $n$ transmitter experiments and a subscript has been added to each quantity that depends on the specific transmitter being used.

Functional (12) is minimized by $n$ independent under-determined regularised solutions $q_{i}$ which provide $n$ estimates of $v_{i}$. The dilation function is now given by

$$
h(x)=-\lambda \frac{1}{n} \sum_{i} v_{i}(x) \quad x \in D_{k}
$$

Figure 6 provides an example of a reconstruction for this situation. Figure 6A illustrates a simulated object located $30 \mathrm{~mm}$ above the model of a 6 " display surrounded by 32 electrodes. Each electrode in turn 


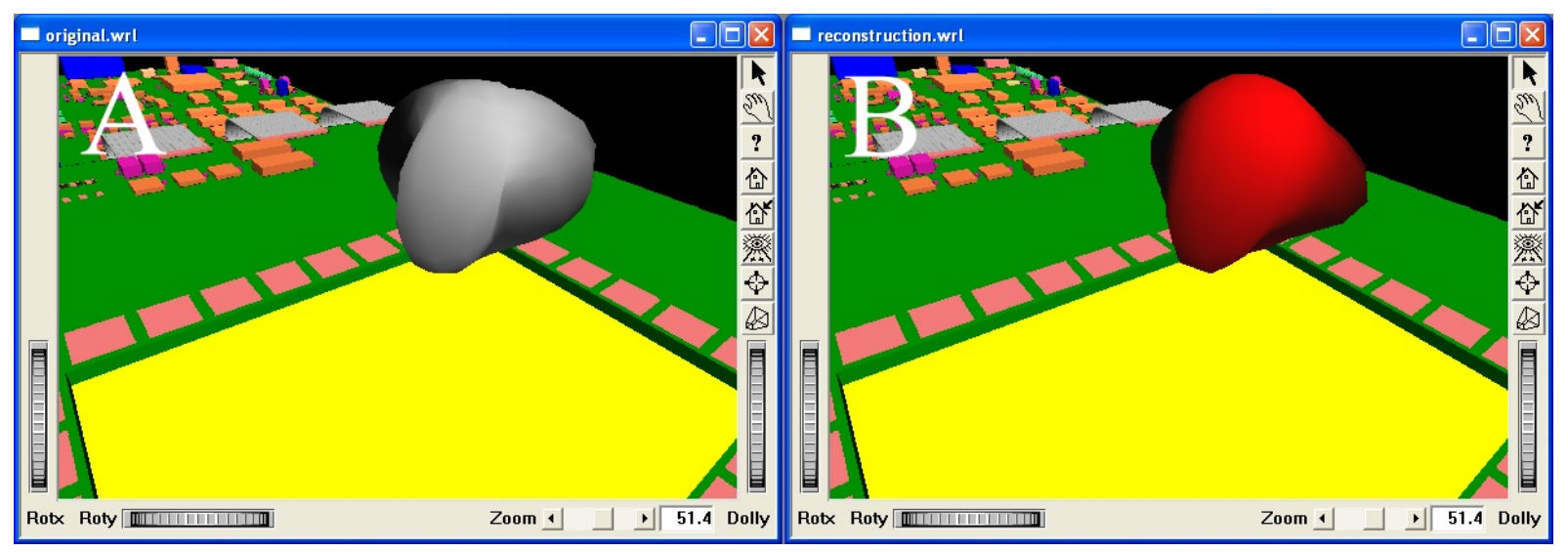

Figure 6. Perspective views of original (A) and reconstructed object (B) above a model experimental system.

functions as transmitter electrode and all electrodes function as receive electrode. The charge images $c_{i}$ for each transmitter are calculated from (3), after solving (2) using midpoint quadrature on a triangle mesh for the object consisting typically of 420 triangles. Doubling or halving the triangle mesh does not affect the results. The computer code used was developed separately from that used to calculate the results shown in the previous sections. $1 \%$ noise was added to the measurements from the 32 edge electrodes. The white object in figure $6 \mathrm{~A}$ illustrates the original object while the object in figure $6 \mathrm{~B}$ illustrates the reconstructed object. An intial guess obtained from Gauss-Newton optimization of the position and size of a sphere, was used as initial guess and 12 dilation iterations applied subsequently. Clearly the object is reconstructed in the correct place with some shape features. If the object is moved and rotated above the display, the location and orientation continue be to reconstructed correctlył.

Figure 7 provides more detail on the shape reconstruction. Figure 7A shows cross sections in the $x_{3}=30 \mathrm{~mm}$ plane and 7B shows cross sections in the $x_{2}=0$ plane. The full line illustrates the original object and the dashed lines five reconstructions for different draws of the noise. Regularisation parameters $\hat{\alpha}=0.1$ and $\hat{\lambda}=1$ were used. Behaviour as illustrated in Figure 5 was observed for increased regularisation or noise.

The approach above solves the inverse problems for each charge image $c_{i}$ separately using prior $q_{p, i}=S^{-1} t_{i}$ for each case. An alternative would be a Kacmarz like approach. The Kacmarz approach is an iterative method used, for instance, in Computerized Tomography[29] in which an orthogonal projection of the current solution is applied into the affine subspace of the set of equations provided by the next incident direction. Here we can use the solution $\left(v_{i-1}, q_{i-1}\right)$ for transmitter $i-1$ to provide a prior for the solution $\left(v_{i}, q_{i}\right)$ at transmitter $i$. That is, in (12) we replace $q_{p, i}$ with

$$
q_{K, i}=S^{-1}\left(t_{i}+v_{i-1}\right)
$$

This uses the fact that dilation function (8), of the solution $\left(v_{i-1}, q_{i-1}\right)$ should be identical to the dilation function of the next solution $\left(v_{i}, q_{i}\right)$. Hence rather than using $v_{i}=0$ as prior information, we use $v_{i}=v_{i-1}$ instead for $i \geq 2$. For $i=1$ the prior $v_{1}=0$ is used. The dilation is now not calculated from a sum as in (13) but only from the last solution. That is, $h=-\lambda v_{n}$. Normalisation of the regularisation as defined in (10) and (11), continues to be used.

Figures 7C and 7D illustrate cross sections of the reconstructed object for this case. Here $\hat{\alpha}=1$ and $\hat{\lambda}=1$ was used.

\section{CONCLUSIONS}

In this paper we have presented the problem of finding the shape of an earthed object from electrostatic measurements made in a plane. The problem is ill-posed but we have shown that an iterative algorithm with three levels of regularisation can recover shape information from noisy data. The algorithm is regularised by 1) the assumption that the initial guess is close to the real object, 2) by the relaxation

$\ddagger$ Note to the reviewers and editor. Movies in animated gif format of all results are available at http://www.ma.umist.ac.uk/bl/objrecon/ 

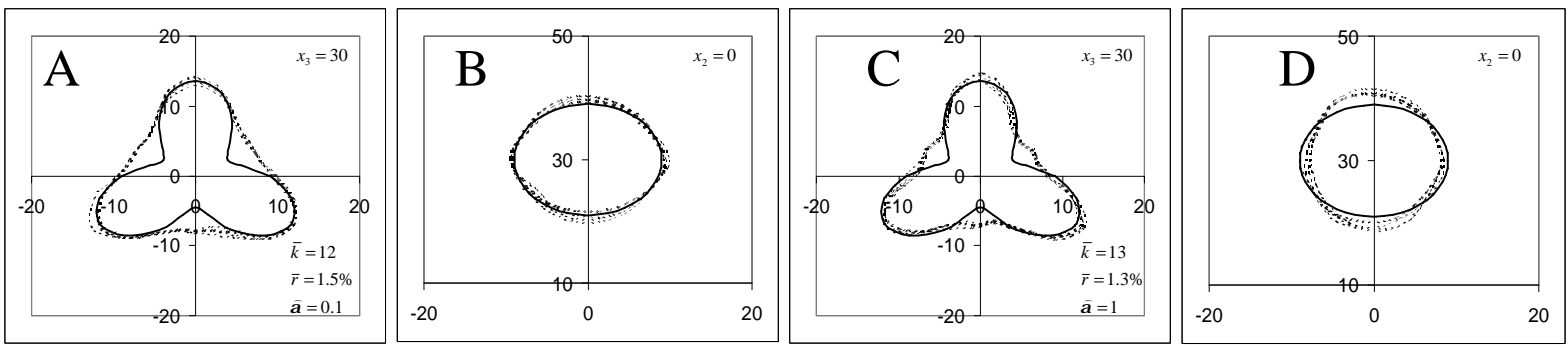

Figure 7. Cross sections of the orginal (bold) and reconstructed object (dashed) 3D object from edge capacitance data with multiple transmitters. A and B: using (13), C and D: using (14)

parameter in the deformation of the guess and 3) by the stopping criterion. We have shown that a simplification can be made to the minimization functional, which yields similar reconstruction results but makes the numerical execution three times faster. We have introduced a scaling of the regularisation parameters that makes the algorithm robust across a range of object and sensor sizes, as well as different discretization levels.

The reconstruction works for realistic sensor configurations.

[1] Cees van Berkel. 3d touchless display interaction. In SID Proc Int Symp, vol 33, number 2, pp1410-1413, May 19-24, 2002.

[2] Thomas G Zimmerman, Joshua R Smith, Joseph A Paradiso, David Allport, and Neil Gershenfeld. Applying electric field sensing to human-computer interfaces. CHI95, Human Factors in Computing Systems, ACM Press pp280-287, 1995.

[3] John David Jackson. Classical Electrodynamics. John Wiley Sons, New York, 2nd edition, 1975.

[4] Kasper van Wijk. Answers to a selection of problems from Jackson's Classical Electrodynamics. Samizdat Press, Golden, Colorado, samizdat.mines.edu, 1996.

[5] Peter Hähner. An inverse problem in electrostatics. Inverse Problems vol 15 pp961-975, 1999.

[6] Ibrahim Akduman and Rainer Kress. Electrostatic imaging via conformal mapping. Inverse Problems vol 18 pp1659$1672,2002$.

[7] O-P Tossavainen and M Vauhkonen. Estimating shapes and free surfaces with electrical impedance tomography. Proc 3rd World Congress on Industrial Process Tomography, Banff, Canada, 2003.

[8] M McIver. Characterisation of surface breaking cracks in metal sheets by using ac electric fields. Proc R Soc A vol 421 179-194, 1989.

[9] N D Aparicio and M K Pidcock. The boundary inverse problem for the laplace equation in two dimensions. Inverse Problems vol 12 pp565-577, 1996.

[10] E Beretta and S Vessella. Stable determination of boundaries from cauchy data. SIAM J Math Anal vol 30 pp220-232, 1998.

[11] G Alessandrini and L Rondi. Optimal stability for the inverse problem of multiple cavities. Journal of Differential Equations vol 176 pp356-386, 2001.

[12] J Cheng, Y C Hon, and M Yamamoto. Conditional stability estimation for an inverse boundary problem with nonsmooth boundary in $r^{3}$. Trans. Amer. Math. Soc. vol 353 pp4124-4138, 2001.

[13] C G Xie, S M Huang, B S Hoyle, R Thorn, C Lenn, D Snowden, and M S Beck. Electrical capacitance tomography for flow imaging: system model for development of image reconstruction algorithms and design of primary sensors. IEEE Proceedings-G vol 139 pp89-98, 1992.

[14] N D Young, G Harkin, R M Bunn, D J McCulloch, R W Wilks, and A G Knapp. Novel fingerpring scanning arrays using polysilicon tfts on glass and polymer substrates. IEEE Electron Devices vol 14 pp19-20, 1997.

[15] T. Bach, C.R. Chatwin, and R.C.D. Young. Capacitive sensor arrays - digitising objects in three dimensions. Proceedings Proc. Int. Conf. in Sensors and Transducers (MTEC 1999), NEC Birmingham UK, 17th-18th Februar, 1997.

[16] Hal Philipp. Charge transfer sensing. Sensor-Review (UK), vol.19 pp96-105, 1999.

[17] C van Berkel and W R B Lionheart. Reconstruction of a grounded object in an electrostatic halfspace with an indicator function. Submitted to Inverse Problems in Science and Engineering, 2005.

[18] Sungwhan Kim, Ohin Kwon, and Jin Keun Seo. Location search techniques for a grounded conductor. SIAM J Appl Math vol 62 pp1383-1393, 2002.

[19] Gerald B Folland. Introduction to Partial Differential Equations. Princeton University Press, Princeton, 1995.

[20] J Kaipio, V Kolehmainen, E Somersalo, and M Vauhkonen. Statistical inversion and monte carlo sampling methods in electrical impedance tomography. Inverse Problems vol 16 pp1487-1522, 2000.

[21] David Colton and Rainer Kress. Inverse Acoustic and Electromagnetic Scattering Theory. Springer-Verlag, New York, 1998.

[22] A Kirsch and R Kress. An optimisation method in inverse acoustic scattering. in Boundary Elements IX, C Brebbia, W Wendland and G Kuhn eds, Springer-Verlag, Heidelberg pp3-18, 1987.

[23] F Santosa. A level-set approach for inverse problems involving obstacles. ESAI: COCV vol 1 17-33, 1996.

[24] Oliver Dorn, Eric L Miller, and Carey M Rappaport. A shape reconstruction method for electromagnetic tomography using adjoint fields and level sets. Inverse Problems vol 16 pp1119-1156, 2000.

[25] A Leitao and O Scherzer. On the relation between constraint regularisation, level sets, and shape optimization. Inverse 
Problems vol 19 ppL1-L11, 2003

[26] C A Brebbia and S Walker. Boundary Element Techniques in Engineering. Butterworth, London, 1st edition, 1980.

[27] Federico París and José Ca nas. Boundary Element Method, Fundamentals and Applications. Oxford University Press, Oxford, 1st edition, 1997.

[28] Andreas Karageorghis and Graeme Fairweather. The method of fundametal solutions for axisymmetric potential problems. Int J Numer Meth Engng vol 44 pp1653-1669, 1999.

[29] Frank Natterer. The Mathematics of Computerized Tomography. John Wiley Sons, Stuttgart, 1986. 